\title{
Solution of heat removal from nuclear reactors by natural convection
}

\author{
Pavel Zitek ${ }^{1, a}$ and Vaclav Valenta ${ }^{1}$ \\ ${ }^{1}$ UWB in Pilsen, Department of Power System Engineering, Univerzitni 8, 30614 Pilsen, Czech Republic
}

\begin{abstract}
This paper summarizes the basis for the solution of heat removal by natural convection from both conventional nuclear reactors and reactors with fuel flowing coolant (such as reactors with molten fluoride salts MSR).The possibility of intensification of heat removal through gas lift is focused on. It might be used in an MSR (Molten Salt Reactor) for cleaning the salt mixture of degassed fission products and therefore eliminating problems with iodine pitting. Heat removal by natural convection and its intensification increases significantly the safety of nuclear reactors. Simultaneously the heat removal also solves problems with lifetime of pumps in the primary circuit of high-temperature reactors.
\end{abstract}

\section{Introduction}

Natural convection and its intensification using gas-lift is one of the inherent and passive safety devices discussed in [3]. They are required for new types of 4th generation nuclear reactors. [1]. Documentation for designs of natural convection and gas-lift systems for conventional nuclear reactors is missing from Czech literature and for the selected type of MSR with flowing fuel-coolant mixture of fluoride salts the methodology is not even elaborated in the English literature.

\section{Model of calculation of primary circuit for natural convection}

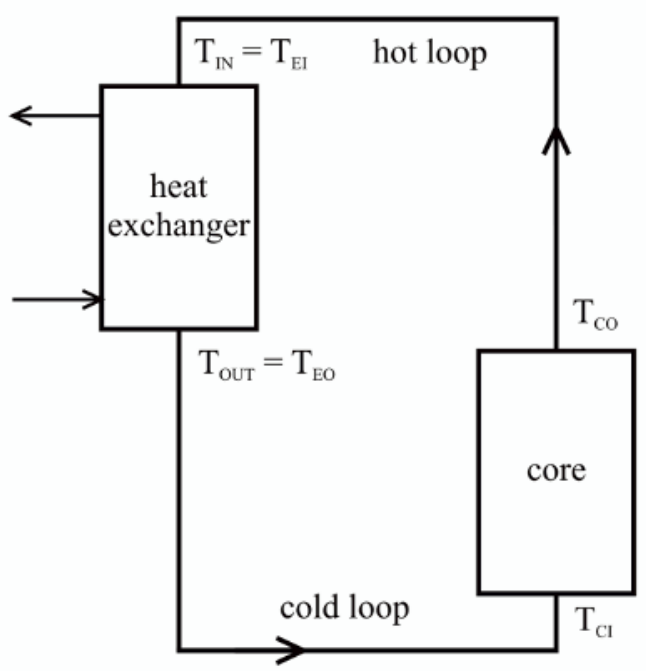

Figure 1
First, we derive formulas for a simplified diagram of the primary circuit of a conventional reactor, where the coolant passes through the primary circuit and heat is produced only in fuel cells in the active core.

Our model will comprise section $i$ :

$\begin{array}{ll}i=1 & \text { core (index } C \text { ) } \\ i=2 & \text { hot loop (index } H L) \\ i=3 & \text { heat exchanger (index } E \text { ) } \\ i=4 & \text { cold loop (index } C L \text { ) } \\ \text { out } & \text { index } O \\ \text { in } & \text { index } I\end{array}$

For individual parts we can write the equations:

$$
\begin{aligned}
& M_{C} \cdot c_{p} \cdot \frac{d \overline{T_{C}}}{d t}=W \cdot c_{p} \cdot\left[T_{C O}-T_{C I}\right]=P_{C} \\
& M_{H L} \cdot c_{p} \cdot \frac{d \overline{T_{H L}}}{d t}=W \cdot c_{p} \cdot\left[T_{E I}-T_{C O}\right] \\
& M_{E} \cdot c_{p} \cdot \frac{d \overline{T_{E}}}{d t}=W \cdot c_{p} \cdot\left[T_{E O}-T_{E I}\right] \\
& M_{C L} \cdot c_{p} \cdot \frac{d \overline{T_{C L}}}{d t}=W \cdot c_{p} \cdot\left[T_{C I}-T_{E O}\right] \\
& M_{i}=\rho\left(\bar{T}_{l}\right) \cdot V_{i}
\end{aligned}
$$

where:

$$
\begin{array}{lll}
P_{C} & {[\mathrm{~W}]} & \text { thermal output of reactor } \\
c_{p} & {[\mathrm{~J} / \mathrm{kg} \cdot \mathrm{K}]} & \text { specific heat capacity } \\
M_{i} & {[\mathrm{~kg}]} & \text { mass of coolant in section } i \\
\rho & {\left[\mathrm{kg} / \mathrm{m}^{3}\right]} & \text { density of coolant at mean temperature } \\
V_{i} & {\left[\mathrm{~m}^{3}\right]} & \text { volume of section } i \\
& \quad M_{C}+M_{H L}+M_{E}+M_{C L}=M_{T}
\end{array}
$$

$M_{T} \quad[\mathrm{~kg}] \quad$ total weight of the primary coolant under steady operation (ignoring weight of coolant in the pressurizer, and in steady operation is not involved in heat transfer)

\footnotetext{
${ }^{\mathrm{a}}$ Corresponding author: zitek@kke.zcu.cz
} 
$W \quad[\mathrm{~kg} / \mathrm{s}] \quad$ mass of coolant flow in individual $i$ sections

$$
W=W_{i}=\rho_{i} \cdot c_{i} \cdot A_{i}
$$

where:

$\begin{array}{lll}c_{i} & {[\mathrm{~m} / \mathrm{s}]} & \text { speed of coolant flow in section } i \\ A_{i} & {\left[\mathrm{~m}^{2}\right]} & \text { flow section in section } i\end{array}$

Assuming steady flow and heat transfer in exchanger $W=W_{\infty}$ and ignoring losses in hot and cold loops is valid $T_{C O}=T_{V I}$ a $T_{V O}=T_{C I}$, therefore:

$$
\frac{d \overline{T_{H L}}}{d t}=0 \mathrm{a} \frac{d \overline{T_{C L}}}{d t}=0
$$

Equations (1) and (3) remain for the solution.

\section{Derived formulae for natural convection of coolant in conventional nuclear reactors with fuel cells}

Natural convection occurs when the forces of buoyancy overcome resistance forces (loss forces), inertia and gravity. For incompressible fluids it is based on the solution of the continuity equation

$$
\dot{V}=\overline{c_{1}} \cdot A_{1}=\overline{c_{2}} \cdot A_{2}
$$

where

$$
\begin{array}{lll}
\dot{V} & {\left[\mathrm{~m}^{3} / \mathrm{s}\right]} & \text { is flow volume } \\
\overline{c_{l}} & {[\mathrm{~m} / \mathrm{s}]} & \text { is average speed in section } i
\end{array}
$$

And the Bernoulli equation, modified to a form suitable for individual sections of the model.

$$
\begin{gathered}
\Delta p=p_{1}-p_{2}=\rho \cdot e_{\text {dis }}+\rho \cdot \frac{\bar{c}_{2}^{2}-\bar{c}_{1}^{2}}{2}+\rho \cdot g \cdot \\
\cdot\left(z_{2}-z_{1}\right)
\end{gathered}
$$

where

$g \quad\left[\mathrm{~m} / \mathrm{s}^{2}\right] \quad$ gravitational acceleration

$z_{i} \quad[\mathrm{~m}] \quad$ vertical coordinates in section $i$

$e_{\text {dis }}\left[\mathrm{m}^{2} / \mathrm{s}^{2}\right]$ the sum of all local and frictional pressure losses in section $i$

For local pressure losses the following relation applies:

$$
e_{\text {dis } j}=\xi_{j} \cdot \frac{\bar{c}_{j}^{2}}{2}
$$

where $\xi_{j}$ is loss coefficient $j$-th type.

For friction pressure losses the following relation applies :

$$
e_{\text {dis } j}=\lambda_{i} \cdot \frac{L_{i}}{D_{e i}} \mid D_{e i}=\frac{4 \cdot A_{i}}{o}
$$

where

$\lambda_{i}[1]$ is friction coefficient dependant on surface roughness in section $i$
$L_{i} \quad[\mathrm{~m}]$
$D_{e i} \quad[\mathrm{~m}]$
is length of piping in section $i$
$o \quad[\mathrm{~m}]$
is equivalent diameter
is wetted perimeter

For closed circuit (e.g. primary circuit) is valid:

$$
\sum_{i} \Delta p_{i}=0
$$

From equations for natural convection of coolant in the primary circuit we easily obtain the equation for balanced flow of coolant $\mathrm{W}_{\infty}$.

$\tilde{\chi}_{p r} \cdot \frac{W_{\infty}^{2}}{2 \cdot \bar{\rho}_{p r}}+g \cdot(\bar{\rho} \cdot \Delta z)_{p r}=0 \quad \mid \tilde{\chi}_{p r}=\chi_{p r} / \overline{A^{2}}$

where $\tilde{\chi}_{p r}\left[1 / \mathrm{m}^{2}\right]$ is total effective loss coefficient in primary

$$
\frac{1}{\bar{A}}=\frac{\bar{\rho}}{L} \cdot \int_{x_{0}}^{x_{L}} \frac{d x}{\rho(x) \cdot A(x)}
$$

$\bar{\rho}_{i}$ the mean specific density of coolant in the $\mathrm{i}$-th segment of of the primary

$$
\bar{\rho}=\frac{1}{V} \cdot \int_{x_{0}}^{x_{L}} \rho(x) \cdot A(x) \cdot d x
$$

For balanced flow is valid:

$$
W_{\infty}=\rho_{i} \cdot c_{i} \cdot A_{i}
$$

$\bar{\rho}$ is the mean specific density

$$
\bar{\rho}=\frac{1}{V} \cdot \int_{x_{0}}^{x_{L}} \rho(x) \cdot A(x) \cdot d x
$$

From(12)

$$
\begin{array}{lll}
c & {[\mathrm{~m} / \mathrm{s}]} & \text { is flow speed } \\
A & {\left[\mathrm{~m}^{2}\right]} & \text { is flow section } \\
g & {\left[\mathrm{~m} / \mathrm{s}^{2}\right]} & \text { is gravitational acceleration } \\
\Delta z & {[\mathrm{~m}]} & \text { is vertical change in primary }
\end{array}
$$

For expressing loss we must determine positional changes and density of coolant in each segment $i$ for coolant $\rho_{i}(T)$.

$$
\begin{gathered}
\bar{\rho}_{i}=\rho\left(T_{\max }^{i}\right)-\frac{\rho\left(T_{\max }^{i}\right)-\rho\left(T_{\min }^{i}\right)}{2} \\
\left(\bar{\rho}_{i} \cdot \Delta z\right)_{\operatorname{Pr}}=\rho \cdot\left(z_{O}-z_{i}\right)_{C}+\rho \cdot\left(z_{O}-z_{i}\right)_{H L}+ \\
+\rho \cdot\left(z_{O}-z_{i}\right)_{E}+\rho \cdot\left(z_{O}-z_{i}\right)_{C L}
\end{gathered}
$$

$z$ are vertical coordinates in segments $\left(z_{O}\right.$ at outputs and $z_{I}$ at inputs of coolant to segment)

First, we make the assumption that changes in heat and thus in the temperature only occur in the core and the exchanger and there the density changes linearly.

Relation (19) we re-write in the form:

$$
\begin{gathered}
\left(\bar{\rho}_{i} \cdot \Delta z\right)_{p r}=\rho_{C O} \cdot \\
\cdot\left[\frac{1}{2} \cdot\left(z_{C O}-z_{C I}\right)+\left(z_{H L O}-z_{H L I}\right)+\frac{1}{2} \cdot\right. \\
\left.\cdot\left(z_{E O}-z_{E I}\right)\right]+\rho_{C I} \cdot \\
\cdot\left[\frac{1}{2} \cdot\left(z_{E O}-z_{E I}\right)+\left(z_{C L O}-z_{C L I}\right)+\frac{1}{2}\right. \\
\left.\cdot\left(z_{C O}-z_{C I}\right)\right]
\end{gathered}
$$

If we consider the height of the input of coolant into the segment is the same as the output from the previous segment, is valid:

$z_{H L I}=z_{C O} ; z_{E I}=z_{H L O} ; z_{C I}=z_{E O} ; z_{C I}=z_{C L O}$

Then:

$$
\left(\bar{\rho}_{i} \cdot \Delta z\right)_{p r}=-\left(\rho_{C I}-\rho_{C O}\right) \cdot\left(\bar{z}_{E}-\bar{z}_{C}\right)
$$

term $\left(\rho_{C I}-\rho_{C O}\right)$ can be modified

$$
\left(\rho_{C I}-\rho_{C O}\right)=-\bar{\rho} \cdot \beta \cdot \Delta T_{C}
$$


where

$$
\begin{array}{ccc}
\rho_{C I}=f\left(T_{C I}\right) & \bar{z}_{E}=\frac{z_{E I}+z_{E O}}{2} & T_{C I}=T_{I N} \\
\rho_{C O}=f\left(T_{C O}\right) & \bar{z}_{C}=\frac{z_{C I}+z_{C O}}{2} & T_{C O}=T_{O U T}
\end{array}
$$

$\bar{z}_{i} \quad[\mathrm{~m}] \quad$ are mean vertical coordinates in segment

$\begin{array}{lll}i & & \\ \bar{\rho}_{C} & {\left[\mathrm{~kg} / \mathrm{m}^{3}\right]} & \text { is mean density of coolant in core }\end{array}$

$$
\begin{array}{ll}
\bar{\rho}_{C} \quad\left[\mathrm{~kg} / \mathrm{m}^{3}\right] & \text { is mean density of coolant } \\
& \bar{\rho}_{C}=\frac{\rho\left(T_{C O}\right)+\rho\left(T_{C I}\right)}{2}
\end{array}
$$

$\beta[1 / \mathrm{K}]$ is the coefficient of volumetric expansion

$\Delta T_{C}[\mathrm{~K}] \quad$ is temperature difference of coolant when passing through zone

$$
\Delta T_{C}=\left(T_{C I}-T_{C O}\right)
$$

$\bar{z}_{E}, \bar{z}_{C}[\mathrm{~m}]$ the mean height of the heat exchanger and zones

After inserting into (13) we obtain:

$$
\begin{aligned}
& \tilde{\chi}_{p r} \cdot \frac{W_{\infty}^{2}}{2 \cdot \bar{\rho}}-\bar{\rho} \cdot \beta \cdot g \cdot\left(\bar{z}_{E}-\bar{z}_{C}\right) \cdot \Delta T_{C}=0 \\
& \text { Pro }
\end{aligned}
$$

$$
\Delta T_{C}=\frac{P}{c_{p} \cdot W_{\infty}}
$$

where $P[\mathrm{~W}]$ is the total thermal power of core. After substitution and modifications we get:

$$
\begin{aligned}
& \tilde{\chi}_{p r} \cdot \frac{W_{\infty}^{3}}{2 \cdot \bar{\rho}}-\bar{\rho} \cdot \beta \cdot g \cdot\left(\bar{z}_{E}-\bar{z}_{C}\right) \cdot \frac{P}{c_{p}}=0 \\
& \quad W_{\infty}=\left[\frac{2 \cdot \bar{\rho}^{2} \cdot \beta \cdot g \cdot P}{\tilde{\chi}_{p r} \cdot c_{p}} \cdot\left(\bar{z}_{E}-\bar{z}_{C}\right)\right]^{1 / 3} \\
& \text { or }
\end{aligned}
$$

$$
\begin{gathered}
\Delta T_{C}=\left(\frac{P}{c_{p} \cdot \bar{\rho}}\right)^{2 / 3} \cdot\left[\frac{\tilde{\chi}_{p r}}{2 \cdot \beta \cdot g \cdot\left(\bar{z}_{E}-\bar{z}_{C}\right)}\right]^{1 / 3} \\
P=\frac{c_{p} \cdot \tilde{\chi}_{p r} \cdot W_{\infty}^{3}}{2 \cdot \bar{\rho}^{2} \cdot g \cdot \beta \cdot\left(z_{E}-z_{C}\right)}
\end{gathered}
$$

From relations (26) to (28) it can be seen that the exchanger must be located above the active core to achieve natural convection and divert power from the core, including the residual power (up to about $7 \%$ of the area) from the shutdown reactor.

The basic problem is the accuracy of the determination of total losses in the primary. One of the components is friction loss, which strongly depends on the Reynolds similarity number and roughness of piping. Here we prepare the dependence of the Reynolds number on the characteristics of natural convection $W_{\infty}$.

$$
\mathrm{Re}=\frac{D_{e k v} \cdot c}{v} \cdot \frac{\rho}{\rho}=\frac{D}{\eta \cdot A} \cdot W_{\infty}
$$

I we consider $D_{e k v}=4 \cdot A / o$, then we get

$$
\operatorname{Re}=\frac{4 \cdot W_{\infty}}{\eta \cdot o}
$$

where:

$$
\begin{array}{lll}
v & {\left[\mathrm{~m}^{2} / \mathrm{s}\right]} & \text { is kinematic viscosity } \\
o & {[\mathrm{~m}]} & \text { is wetted perimeter } \\
\eta & {[\mathrm{kg} / \mathrm{m} \cdot \mathrm{s}]} & \text { is dynamic viscosity }
\end{array}
$$

For insertion into (31) it was considered that $\eta=v \cdot \rho$ and $W_{\infty}=\rho \cdot c \cdot A$.

To determine the Reynolds number in individual parts of the primary we must take into account that dynamic viscosity $\eta$ is heat dependent $\left(\eta=\eta\left(T_{i}\right)\right)$ and refine individual parts of total losses in the primary.

When using $\mathrm{H}_{2} \mathrm{O}$ as a coolant, it is necessary to consider the possibility of the coolant boiling during natural convection. A two-phase flow arises (water + steam) and it is necessary to determine the loss in this case. This is common for BWR (Boiling Water Reactors).

Extending the methodology derived here to other sections such as e.g. the primary coolant inlet chamber below the active zone and the outlet chamber above it and the transition to chimney draft, etc. does not constitute a problem, as relations (25) to (28) remain valid. It is only necessary to include the loss of these sections in the total loss coefficient.

\section{Natural convection in systems with flowing fuel}

The above method is unsuitable for shut down reactors with a flowing fuel mix because of the evolution of residual heat in the whole volume of the primary. For this type of reactor, the neglected heat evolution in other parts of the primary is a very rough approximation.

In conventional reactors with solid fuel residual power is concentrated in fuel cells. The main advantage of reactors with molten fluoride salts (MSR - Molten Salt Reactor) is the ability to clean the mix from fission products (FP) during operation. This is done in two ways:

a) By bubbling the mixture with $\mathrm{He}$ and the removal of gaseous $\mathrm{FP}$ such as $\mathrm{Kr}$, $\mathrm{Xe}$ and tritium and removing the He by cleaning. This method virtually eliminates the so called "iodine pit".

b) Removal of part of the fuel mix and chemical cleaning done at the nuclear power plant. Depending on frequency and quantity it is possible to substantially reduce residual evolution of heat from FP.

The method for solving the balance of FP during cleaning can be found in the works [6,7].

The presence of actinides in the fuel-coolant mix causes the formation of an independent source of neutrons (from spontaneous fission and from $(\alpha, n)$ reactions on the salt elements). Other neutrons originate from FP as so-called 'delayed neutrons'.

This independent source of neutrons in the fuel mix causes additional heat generation in all parts of the primary outside the core due to fission in its otherwise subcritical parts.

This means that the design of the primary must be tested at sub-criticality in its individual sections. The source of neutrons is according to size $k_{e f}$ further multiplied and causes heat generation (similar to the case in the active core) in every part of the primary. It is 
therefore necessary to expand the formulation of the previously solved problem.

First we modify relations(1) to (4):

$$
\begin{gathered}
M_{C} \cdot c_{p} \cdot \frac{d \overline{T_{C}}}{d t}=W \cdot c_{p} \cdot\left[T_{C O}-T_{C I}\right]=P_{C} \\
M_{H L} \cdot c_{p} \cdot \frac{d \overline{T_{H L}}}{d t}=W \cdot c_{p} \cdot\left[T_{E I}-T_{C O}\right]=\Delta P_{H L} \\
M_{E} \cdot c_{p} \cdot \frac{d \overline{T_{E}}}{d t}=W \cdot c_{p} \cdot\left[T_{E O}-T_{E I}\right]=\Delta P_{E}-Q \\
M_{C L} \cdot c_{p} \cdot \frac{d \overline{T_{C L}}}{d t}=W \cdot c_{p} \cdot\left[T_{C I}-T_{E O}\right]=\Delta P_{C L}
\end{gathered}
$$

where

\section{$Q \quad[\mathrm{~W}]$}

$P_{C} \quad[\mathrm{~W}]$

$\Delta P_{H L}[\mathrm{~W}]$

$\Delta P_{E}[\mathrm{~W}]$

$\Delta P_{C L}[\mathrm{~W}]$

$$
\text { is power convected to secondary }
$$
is total thermal power of reactor is power released in hot loop is power produced in heat exchanger

$$
Q=\alpha \cdot A \cdot \Delta T_{m}=\left(P_{C}+\Delta P_{H L}+\Delta P_{E}+\Delta P_{C L}\right)
$$

For reactors with flowing fuel the power produced in individual parts of the primary $i$ will generally be varied and determined by the sum

$$
P_{i}=P_{F P}+P_{Q n}+P_{a c t}
$$

where

$P_{F P} \quad[\mathrm{~W}] \quad$ is given by the energy from decay of $\beta$ and $\gamma$ FP and depends on the mode of cleaning the fuel mixture from $\mathrm{FP}$

$P_{\text {act }}$ [W] is given by the energy from decay of $\beta$ and $\gamma$ actinide and its spontaneous fission

$P_{Q n} \quad[\mathrm{~W}] \quad$ is determined by actinide fission from an external source of neutrons (which has three components) in different subcritical parts of the primary s $k_{e f}<1$

$$
Q_{n}^{i}=Q_{n}^{s f}+Q_{(\alpha, n)}+Q_{\text {deloy } n}
$$

where

$Q_{n}^{s f}[\mathrm{n} / \mathrm{s}] \quad$ is source of neutrons from spontaneous fission of actinide

$Q_{(\alpha, n)}[\mathrm{n} / \mathrm{s}] \quad$ is source of neutrons arising with the help of $(\alpha, n)$ reactions, where $\alpha$ are emitted during $\alpha$ decay of actinide and their reaction with elements in the fluoride salts.

$Q_{\text {deloy n }}[\mathrm{n} / \mathrm{s}]$ is source of neutrons from FP (delayed neutrons), here there is a strong dependency on time of leaving the core and power

For subcritical area is also valid:

where

$$
P_{Q n}^{i}=k \cdot E_{f} \cdot \frac{Q_{n}^{i}}{\left(1-k_{e f}^{i}\right)}
$$

$E_{f} \quad[\mathrm{MeV}] \quad$ is energy released during fission

$k \quad[\mathrm{~W} / \mathrm{MeV}]$ conversion factor $\mathrm{z}[\mathrm{MeV}]$ on $[\mathrm{W}]$

$Q_{n}^{i} \quad[\mathrm{MeV} / \mathrm{s}] \quad$ is total source of neutrons in part $i$ of the primary s $k_{\text {ef }}^{i}<1$ (effective multiplication coefficient $i$ th part). It will be advantageous to consider conditions $k_{\text {ef }}^{i}<0.98$ and perform a study of the influence with even smaller $k_{\text {ef }}^{i}$.

For a certain degree of simplification it is possible to suppose that during a selected cleaning regime the variables $P_{\check{S} P}, P_{a c t}, Q_{n}^{s f}, Q_{(\alpha, n)}$ change slowly and are constant during their circulation in the fuel-coolant mix. $Q_{\text {deloy n }}$ from delayed neutrons can be ignored during reactor shutdown. During reactor operation, it is possible to use groups of delayed neutrons with effective decay constants $\lambda_{g}\left[s^{-1}\right]$.

The neutron source can be determined using the program SOURCE 4C (RSIC Code Package CCC-661). Results from calculation of $(\alpha, n)$ reactions to the salt mix and neutron spectrum can be obtained from JENDL $(\alpha, n)$ Reaction Data File 2005 (viz also Murata T: JAERI-Conf 98-003 p 215 and [10, 11, 21], where resonance profiles of reactions $(\alpha, n)$ are considered. It would be appropriate to compare the calculation results from both sources.

We break down the balance $\Delta p$ for each part of the primary. We assume that we will use identical exchangers arranged in parallel in the integral layout N.

For each part of the primary we obtain:

$$
\begin{gathered}
\Delta p_{C}=\left(\frac{L}{A}\right)_{C} \cdot \frac{d W}{d t}+\bar{\chi}_{C} \cdot \frac{W^{2}}{2 \cdot \bar{\rho}_{C}}+g \cdot(\bar{\rho} \cdot \Delta z)_{C} \\
\Delta p_{H L}=\left(\frac{L}{A}\right)_{H L} \cdot \frac{d W}{d t}+\bar{\chi}_{H L} \cdot \frac{W^{2}}{2 \cdot \bar{\rho}_{H L}}+g \cdot \\
\Delta p_{E}=\left(\frac{L}{A}\right)_{E} \cdot \frac{d W}{d t}+\bar{\chi}_{E} \cdot \frac{W^{2}}{2 \cdot \bar{\rho}_{E}}+g \cdot(\bar{\rho} \cdot \Delta z)_{E} \\
\Delta p_{C L}=\left(\frac{L}{A}\right)_{C L} \cdot \frac{d W}{d t}+\bar{\chi}_{C L} \cdot \frac{W^{2}}{2 \cdot \bar{\rho}_{C L}}+g \cdot \\
\cdot(\bar{\rho} \cdot \Delta z)_{C L}
\end{gathered}
$$

For the primary is valid:

$$
\begin{gathered}
\Delta p_{C}+\Delta p_{H L}+\Delta p_{E}+\Delta p_{C L}=0 \\
W=N \cdot W_{E}=W_{C}=W_{H L}=W_{C L}
\end{gathered}
$$

Kirhof hydrodynamic laws also apply.

By combining relations (40) to (43) with relations (44) and (45) we obtain for our design of the primary:

$$
\begin{gathered}
\left(\frac{L}{A}\right)_{p r} \cdot \frac{d W}{d t}+\tilde{\chi}_{p r} \cdot \frac{W^{2}}{2 \cdot \bar{\rho}_{p r}}+g \cdot(\bar{\rho} \cdot \Delta z)_{p r}=0 \\
\text { where: } \\
\left(\frac{L}{A}\right)_{p r}=\left[\left(\frac{L}{A}\right)_{C}+\left(\frac{L}{A}\right)_{H L}+\frac{1}{N} \cdot\left(\frac{L}{A}\right)_{E}+\left(\frac{L}{A}\right)_{C L}\right] \\
\tilde{\chi}_{p r}=\left[\tilde{\chi}_{C}+\tilde{\chi}_{H L}+\tilde{\chi}_{E}+\tilde{\chi}_{C L}\right] \\
\bar{\rho}_{p r}=\left[\bar{\rho}_{C}+\bar{\rho}_{H L}+\bar{\rho}_{E}+\bar{\rho}_{C L}\right] \\
(\bar{\rho} \cdot \Delta z)_{p r}=\left[(\bar{\rho} \cdot \Delta z)_{C}+(\bar{\rho} \cdot \Delta z)_{H L}+(\bar{\rho} \cdot \Delta z)_{E}\right. \\
\left.+(\bar{\rho} \cdot \Delta z)_{C L}\right]
\end{gathered}
$$

where:

$$
\begin{gathered}
\Delta z_{j}=\left(z_{O j}-z_{I j}\right) \\
\bar{\rho}_{j}=\rho\left(\bar{T}_{j}\right)=\frac{\rho\left(\bar{T}_{I j}\right)+\rho\left(\bar{T}_{O j}\right)}{2}
\end{gathered}
$$

For a steady flow is valid $\frac{d W}{d t}=0$ and from equation (46) we obtain 


$$
\frac{\tilde{\chi}_{p r}}{\bar{\rho}_{p r}} \cdot \frac{W_{\infty}^{2}}{2}+g \cdot(\bar{\rho} \cdot \Delta z)_{p r}=0
$$

where $(\bar{\rho} \cdot \Delta z)_{p r}$ is determined from relation (50)

$$
\frac{\tilde{\chi}_{p r}}{\bar{\rho}_{p r}}=\left[\frac{\tilde{\chi}_{C}}{\bar{\rho}_{C}}+\frac{\tilde{\chi}_{H L}}{\bar{\rho}_{H L}}+\frac{1}{N^{2}} \cdot \frac{\tilde{\chi}_{E}}{\bar{\rho}_{E}}+\frac{\tilde{\chi}_{L}}{\bar{\rho}_{C L}}\right]
$$

All empirical values of loss coefficients were measured during steady flow of mix.

$$
\begin{gathered}
W_{\infty}=\left\{\frac{g \cdot \beta \cdot[A+B+C+D]}{c_{p} \cdot\left[\frac{\tilde{\chi}_{C}}{\bar{\rho}_{C}}+\frac{\tilde{\chi}_{H L}}{\bar{\rho}_{H L}}+\frac{1}{N^{2}} \cdot \frac{\tilde{\chi}_{E}}{\bar{\rho}_{E}}+\frac{\tilde{\chi}_{L}}{\bar{\rho}_{C L}}\right]}\right\}^{\frac{1}{3}} \\
A=\bar{\rho}_{C} \cdot P_{C} \cdot\left(z_{O C}-z_{I C}\right) \\
B=\bar{\rho}_{H l} \cdot P_{H L} \cdot\left(z_{O H L}-z_{I H L}\right) \\
C=\bar{\rho}_{E} \cdot P_{E} \cdot\left(z_{O E}-z_{I E}\right) \\
D=\bar{\rho}_{C L} \cdot P_{C L} \cdot\left(z_{O C L}-z_{I C L}\right)
\end{gathered}
$$

Where for $\bar{\rho}_{j}$ is valid (52)

$$
Q=\left[P_{C}+P_{H L}+P_{E}+P_{C L}\right]
$$

We obtain the minimum operating temperature of primary $T_{\min }^{I}$ at the output of the exchanger. For safety reasons this temperature should be at least $100^{\circ} \mathrm{C}$ above the melting point of the salt in use.

\section{Conclusions}

The work gives a basis for determining heat transfer by natural convection in general.

It deals mainly with MSR reactors with flowing fuelcoolant mix, where due to the delayed neutrons and neutrons from $(\alpha, n)$ reactions to light elements of fluoride salts and radiation from fission products lead to production of heat in other subcritical parts of the primary. This production is indeed much lower than in the core, but it affects the temperature distribution in the primary. It also depends on the flow velocity (delayed neutrons).

Suitable pumps that can withstand long-term operation are not available for high temperature MSR. The problem is solved by the intensification of the natural convection using "gas-lift". This theme is discussed in our next article entitled "Parents of Two-Phase Flow, Bubble Distribution and Interfacial Area in Bubble Flow".

\section{Acknowledgements}

The presented research is supported by the project CZ.1.05/2.1.00/03.0108 (Sustainable Energy, SUSEN) and student project SGS-2012-072 (Zvyšování účinnosti, spolehlivosti a životnosti energetických strojů a zařízení 2).

\section{References}

1. V. Valenta, research report KKE 05/2012.

2. V. Valenta, research report KKE 03/2010.
3. V. Valenta, research report KKE 02/2011

4. S.N.Šorin: Sdílení tepla (SNTL Praha 1968)

5. V. Valenta, Modelování a měrení v energetice, 187204 (2010)

6. V. Valenta, research report TECH 03/2002

7. V. Valenta, research report TECH 02/2005 